\title{
On the Smarandache-Pascal derived sequences and some of their conjectures
}

Xiaoxue Li and Di Han*

"Correspondence: handi515@163.com

Department of Mathematics, Northwest University, Xi'an, Shaanxi, P.R. China

\begin{abstract}
For any sequence $\left\{b_{n}\right\}$, the Smarandache-Pascal derived sequence $\left\{T_{n}\right\}$ of $\left\{b_{n}\right\}$ is defined as $T_{1}=b_{1}, T_{2}=b_{1}+b_{2}, T_{3}=b_{1}+2 b_{2}+b_{3}$, generally, $T_{n+1}=\sum_{k=0}^{n}\left(\begin{array}{l}n \\ k\end{array}\right) \cdot b_{k+1}$ for all $n \geq 2$, where $\left(\begin{array}{l}n \\ k\end{array}\right)=\frac{n !}{k ! n-k) !}$ is the combination number. In reference (Murthy and Ashbacher in Generalized Partitions and New Ideas on Number Theory and Smarandache Sequences, 2005), authors proposed a series of conjectures related to Fibonacci numbers and its Smarandache-Pascal derived sequence, one of them is that if $\left\{b_{n}\right\}=\left\{F_{1}, F_{9}, F_{17}, \ldots\right\}$, then we have the recurrence formula $T_{n+1}=49 \cdot\left(T_{n}-T_{n-1}\right), n \geq 2$. The main purpose of this paper is using the elementary method and the properties of the second-order linear recurrence sequence to study these problems and to prove a generalized conclusion.
\end{abstract}

Keywords: Smarandache-Pascal derived sequence; Fibonacci number; combination number; elementary method; conjecture

\section{Introduction}

For any sequence $\left\{b_{n}\right\}$, we define a new sequence $\left\{T_{n}\right\}$ through the following method: $T_{1}=b_{1}, T_{2}=b_{1}+b_{2}, T_{3}=b_{1}+2 b_{2}+b_{3}$, generally, $T_{n+1}=\sum_{k=0}^{n}\left(\begin{array}{l}n \\ k\end{array}\right) \cdot b_{k+1}$ for all $n \geq 2$, where $\left(\begin{array}{l}n \\ k\end{array}\right)=\frac{n !}{k !(n-k) !}$ is the combination number. This sequence is called the Smarandache-Pascal derived sequence of $\left\{b_{n}\right\}$. It was introduced by professor Smarandache in [1] and studied by some authors. For example, Murthy and Ashbacher [2] proposed a series of conjectures related to Fibonacci numbers and its Smarandache-Pascal derived sequence; three of them are as follows.

Conjecture 1 Let $\left\{b_{n}\right\}=\left\{F_{8 n+1}\right\}=\left\{F_{1}, F_{9}, F_{17}, F_{25}, \ldots\right\},\left\{T_{n}\right\}$ be the Smarandache-Pascal derived sequence of $\left\{b_{n}\right\}$, then we have the recurrence formula

$$
T_{n+1}=49 \cdot\left(T_{n}-T_{n-1}\right), \quad n \geq 2 .
$$

Conjecture 2 Let $\left\{b_{n}\right\}=\left\{F_{10 n+1}\right\}=\left\{F_{1}, F_{11}, F_{21}, F_{31}, \ldots\right\},\left\{T_{n}\right\}$ be the Smarandache-Pascal derived sequence of $\left\{b_{n}\right\}$, then we have the recurrence formula

$$
T_{n+1}=125 \cdot\left(T_{n}-T_{n-1}\right), \quad n \geq 2 .
$$

Conjecture 3 Let $\left\{b_{n}\right\}=\left\{F_{12 n+1}\right\}=\left\{F_{1}, F_{13}, F_{25}, F_{37}, \ldots\right\},\left\{T_{n}\right\}$ be the Smarandache-Pascal derived sequence of $\left\{b_{n}\right\}$, then we have the recurrence formula

$$
T_{n+1}=324 \cdot\left(T_{n}-T_{n-1}\right), \quad n \geq 2 .
$$

(c) $2013 \mathrm{Li}$ and Han; licensee Springer. This is an Open Access article distributed under the terms of the Creative Commons Attribution License (http://creativecommons.org/licenses/by/2.0), which permits unrestricted use, distribution, and reproduction in any medium, provided the original work is properly cited. 
Regarding these conjectures, it seems that no one has studied them yet; at least, we have not seen any related results before. These conjectures are interesting; they reveal the profound properties of the Fibonacci numbers. The main purpose of this paper is using the elementary method and the properties of the second-order linear recurrence sequence to study these problems and to prove a generalized conclusion. That is, we shall prove the following.

Theorem Let $\left\{X_{n}\right\}$ be a second-order linear recurrence sequence with $X_{0}=u, X_{1}=v, X_{n+1}=$ $a X_{n}+b X_{n-1}$ for all $n \geq 1$, where $a^{2}+4 b>0$. For any positive integer $d \geq 2$, we define the Smarandache-Pascal derived sequence of $\left\{X_{d n+1}\right\}$ as

$$
T_{n+1}=\sum_{k=0}^{n}\left(\begin{array}{l}
n \\
k
\end{array}\right) \cdot X_{d k+1}
$$

Then we have the recurrence formula

$$
T_{n+1}=\left(2+A_{d}+b \cdot A_{d-2}\right) \cdot T_{n}-\left(1+A_{d}+b \cdot A_{d-2}+(-b)^{d}\right) \cdot T_{n-1},
$$

where the sequence $\left\{A_{n}\right\}$ is defined as $A_{0}=1, A_{1}=a, A_{n+1}=a \cdot A_{n}+b \cdot A_{n-1}$ for all $n \geq 1$. In fact this time, the general term is

$$
A_{n}=\frac{1}{\sqrt{a^{2}+4 b}}\left[\left(\frac{a+\sqrt{a^{2}+4 b}}{2}\right)^{n+1}-\left(\frac{a-\sqrt{a^{2}+4 b}}{2}\right)^{n+1}\right] .
$$

Now we take $b=1$, then from our theorem, we may immediately deduce the following three corollaries.

Corollary 1 Let $\left\{X_{n}\right\}$ be a second-order linear recurrence sequence with $X_{0}=u, X_{1}=v$, $X_{n+1}=a X_{n}+X_{n-1}$ for all $n \geq 1$. For any even number $d \geq 2$, we have the recurrence formula

$$
T_{n+1}=\left(2+A_{d}+A_{d-2}\right) \cdot\left(T_{n}-T_{n-1}\right), \quad n \geq 2 .
$$

Corollary 2 Let $\left\{X_{n}\right\}$ be a second-order linear recurrence sequence with $X_{0}=u, X_{1}=v$, $X_{n+1}=a X_{n}+X_{n-1}$ for all $n \geq 1$. For any odd number $d \geq 2$, we have the recurrence formula

$$
T_{n+1}=\left(2+A_{d}+A_{d-2}\right) \cdot T_{n}-\left(A_{d}+A_{d-2}\right) \cdot T_{n-1}, \quad n \geq 2,
$$

where

$$
A_{n}=A_{n}(a)=\frac{1}{\sqrt{a^{2}+4}}\left[\left(\frac{a+\sqrt{a^{2}+4}}{2}\right)^{n+1}-\left(\frac{a-\sqrt{a^{2}+4}}{2}\right)^{n+1}\right] .
$$

It is clear that $F_{n+1}(a)=A_{n}(a)$ is a polynomial of $a$; sometimes, it is called a Fibonacci polynomial, because $F_{n}(1)=F_{n}$ is Fibonacci number, see [3-5].

If we take $a=1, X_{0}=0, X_{1}=1$ in Corollary 1 , then $\left\{X_{n}\right\}=\left\{F_{n}\right\}$ is a Fibonacci sequence. Note that $A_{n}=F_{n+1}, 2+A_{8}+A_{6}=2+F_{9}+F_{7}=2+34+13=49,2+A_{10}+A_{8}=2+F_{11}+F_{9}=$ $2+89+34=125,2+A_{12}+A_{10}=2+F_{13}+F_{11}=2+233+89=324$; from Corollary 1 , we may immediately deduce that the three conjectures above are true. 
If we take $a=2, X_{0}=P_{0}=0, X_{1}=P_{1}=1$ and $P_{n+1}=2 P_{n}+P_{n-1}$ for all $n \geq 1$, then $P_{n}$ are the Pell numbers. From Corollary 1 , we can also deduce the following.

Corollary 3 Let $P_{n}$ be the Pell number. Then for any positive integer $d$ and

$$
T_{n+1}=\sum_{k=0}^{n}\left(\begin{array}{l}
n \\
k
\end{array}\right) \cdot P_{2 d k+1}
$$

we have the recurrence formula

$$
T_{n+1}=\left(2+P_{2 d+1}+P_{2 d-1}\right) \cdot\left(T_{n}-T_{n-1}\right), \quad n \geq 2 .
$$

On the other hand, from our theorem, we know that if $\left\{b_{n}\right\}$ is a second-order linear recurrence sequence, then its Smarandache-Pascal derived sequence $\left\{T_{n}\right\}$ is also a secondorder linear recurrence sequence.

\section{Proof of the theorem}

To complete the proof of our theorem, we need the following.

Lemma Let integers $m \geq 0$ and $n \geq 2$. If the sequence $\left\{X_{n}\right\}$ satisfying the recurrence relations $X_{n+2}=a \cdot X_{n+1}+b \cdot X_{n}, n \geq 0$, then we have the identity

$$
X_{m+n}=A_{n-1} \cdot X_{m+1}+b \cdot A_{n-2} \cdot X_{m}
$$

where $A_{n}$ is defined as $A_{0}=1, A_{1}=a$ and $A_{n+1}=a \cdot A_{n}+b \cdot A_{n-1}$ for all $n \geq 1$, or

$$
A_{n}=\frac{1}{\sqrt{a^{2}+4 b}}\left[\left(\frac{a+\sqrt{a^{2}+4 b}}{2}\right)^{n+1}-\left(\frac{a-\sqrt{a^{2}+4 b}}{2}\right)^{n+1}\right] .
$$

Proof Now we prove this lemma by mathematical induction. Note that the recurrence formula $X_{m+2}=a \cdot X_{m+1}+b \cdot X_{m}, A_{1}=a, A_{0}=1, A_{n+1}=a \cdot A_{n}+b \cdot A_{n-1}$ for all $n \geq 1$. So $X_{m+2}=A_{1} \cdot X_{m+1}+b \cdot A_{0} \cdot X_{m}$. That is, the lemma holds for $n=2$. Since $X_{m+3}=a \cdot X_{m+2}+$ $b \cdot X_{m+1}=a \cdot\left(a \cdot X_{m+1}+b \cdot X_{m}\right)+b \cdot X_{m+1}=\left(a^{2}+b\right) \cdot X_{m+1}+b a \cdot X_{m}=A_{2} \cdot X_{m+1}+b A_{1} \cdot X_{m}$. That is, the lemma holds for $n=3$. Suppose that for all integers $2 \leq n \leq k$, we have $X_{m+n}=$ $A_{n-1} \cdot X_{m+1}+b \cdot A_{n-2} \cdot X_{m}$. Then for $n=k+1$, from the recurrence relations for $X_{m}$ and the inductive hypothesis, we have

$$
\begin{aligned}
X_{m+k+1} & =a \cdot X_{m+k}+b \cdot X_{m+k-1} \\
& =a \cdot\left(A_{k-1} \cdot X_{m+1}+b \cdot A_{k-2} \cdot X_{m}\right)+b \cdot\left(A_{k-2} \cdot X_{m+1}+b \cdot A_{k-3} \cdot X_{m}\right) \\
& =\left(a \cdot A_{k-1}+b \cdot A_{k-2}\right) \cdot X_{m+1}+b \cdot\left(a \cdot A_{k-2}+b \cdot A_{k-3}\right) \cdot X_{m} \\
& =A_{k} \cdot X_{m+1}+b \cdot A_{k-1} \cdot X_{m-1} .
\end{aligned}
$$

That is, the lemma also holds for $n=k+1$. This completes the proof of our lemma by mathematical induction. 
Now, we use this lemma to complete the proof of our theorem. For any positive integer $d$, from the definition of $T_{n}$ and the properties of the binomial coefficient $\left(\begin{array}{l}n \\ k\end{array}\right)$, we have

$$
\begin{aligned}
\left(\begin{array}{c}
n-1 \\
k
\end{array}\right)+\left(\begin{array}{l}
n-1 \\
k-1
\end{array}\right) & =\frac{(n-1) !}{k !(n-1-k) !}+\frac{(n-1) !}{(k-1) !(n-k) !} \\
& =\frac{(n-1) !}{(k-1) !(n-k-1) !}\left(\frac{1}{k}+\frac{1}{n-k}\right)=\left(\begin{array}{l}
n \\
k
\end{array}\right)
\end{aligned}
$$

and

$$
\begin{aligned}
T_{n+1} & =\sum_{k=0}^{n}\left(\begin{array}{l}
n \\
k
\end{array}\right) \cdot X_{d k+1} \\
& =X_{1}+X_{d n+1}+\sum_{k=1}^{n-1}\left(\left(\begin{array}{c}
n-1 \\
k
\end{array}\right)+\left(\begin{array}{c}
n-1 \\
k-1
\end{array}\right)\right) X_{d k+1} \\
& =\sum_{k=0}^{n-1}\left(\begin{array}{c}
n-1 \\
k
\end{array}\right) \cdot X_{d k+1}+\sum_{k=0}^{n-2}\left(\begin{array}{c}
n-1 \\
k
\end{array}\right) \cdot X_{d k+d+1}+X_{d n+1} \\
& =T_{n}+\sum_{k=0}^{n-1}\left(\begin{array}{c}
n-1 \\
k
\end{array}\right) X_{d k+d+1} .
\end{aligned}
$$

From the lemma, we have $X_{d k+d+1}=A_{d} \cdot X_{d k+1}+b \cdot A_{d-1} \cdot X_{d k}$, by (2) and the definition of $T_{n}$, we may deduce that

$$
\begin{aligned}
T_{n+1} & =T_{n}+\sum_{k=0}^{n-1}\left(\begin{array}{c}
n-1 \\
k
\end{array}\right) \cdot\left(A_{d} \cdot X_{d k+1}+b \cdot A_{d-1} \cdot X_{d k}\right) \\
& =T_{n}+A_{d} \cdot \sum_{k=0}^{n-1}\left(\begin{array}{c}
n-1 \\
k
\end{array}\right) \cdot X_{d k+1}+b \cdot A_{d-1} \cdot \sum_{k=0}^{n-1}\left(\begin{array}{c}
n-1 \\
k
\end{array}\right) \cdot X_{d k} \\
& =\left(1+A_{d}\right) \cdot T_{n}+b \cdot A_{d-1} \cdot \sum_{k=0}^{n-1}\left(\begin{array}{c}
n-1 \\
k
\end{array}\right) \cdot X_{d k} .
\end{aligned}
$$

On the other hand, from the lemma, we also have $X_{d k+d}=A_{d-1} \cdot X_{d k+1}+b \cdot A_{d-2} \cdot X_{d k}$, from this and formula (1), we have

$$
\begin{aligned}
\sum_{k=0}^{n-1}\left(\begin{array}{c}
n-1 \\
k
\end{array}\right) \cdot X_{d k} & =X_{0}+X_{d(n-1)}+\sum_{k=1}^{n-2}\left(\begin{array}{c}
n-1 \\
k
\end{array}\right) \cdot X_{d k} \\
& =X_{0}+X_{d(n-1)}+\sum_{k=1}^{n-2}\left(\left(\begin{array}{c}
n-2 \\
k
\end{array}\right)+\left(\begin{array}{c}
n-2 \\
k-1
\end{array}\right)\right) \cdot X_{d k} \\
& =\sum_{k=0}^{n-2}\left(\begin{array}{c}
n-2 \\
k
\end{array}\right) \cdot X_{d k}+\sum_{k=0}^{n-3}\left(\begin{array}{c}
n-2 \\
k
\end{array}\right) \cdot X_{d k+d}+X_{d(n-1)} \\
& =\sum_{k=0}^{n-2}\left(\begin{array}{c}
n-2 \\
k
\end{array}\right) \cdot X_{d k}+\sum_{k=0}^{n-2}\left(\begin{array}{c}
n-2 \\
k
\end{array}\right) \cdot\left(A_{d-1} \cdot X_{d k+1}+b \cdot A_{d-2} \cdot X_{d k}\right) \\
& =\left(1+b \cdot A_{d-2}\right) \cdot \sum_{k=0}^{n-2}\left(\begin{array}{c}
n-2 \\
k
\end{array}\right) \cdot X_{d k}+A_{d-1} \cdot T_{n-1} \cdot
\end{aligned}
$$


From (3), we can also deduce that

$$
T_{n}=\left(1+A_{d}\right) \cdot T_{n-1}+b \cdot A_{d-1} \cdot \sum_{k=0}^{n-2}\left(\begin{array}{c}
n-2 \\
k
\end{array}\right) \cdot X_{d k} .
$$

Now, combining (3), (4) and (5), we may immediately get

$$
\begin{aligned}
T_{n+1} & =\left(1+A_{d}\right) \cdot T_{n}+b \cdot A_{d-1} \cdot\left(\left(1+b \cdot A_{d-2}\right) \cdot \sum_{k=0}^{n-2}\left(\begin{array}{c}
n-2 \\
k
\end{array}\right) \cdot X_{d k}+A_{d-1} \cdot T_{n-1}\right) \\
& =\left(1+A_{d}\right) \cdot T_{n}+b \cdot A_{d-1}^{2} \cdot T_{n-1}+\left(1+b \cdot A_{d-2}\right) \cdot\left(T_{n}-\left(1+A_{d}\right) \cdot T_{n-1}\right)
\end{aligned}
$$

or equivalent to

$$
\begin{aligned}
T_{n+1} & =\left(2+A_{d}+b \cdot A_{d-2}\right) \cdot T_{n}-\left(1+A_{d}+b \cdot A_{d-2}+b \cdot A_{d} \cdot A_{d-2}-b \cdot A_{d-1}^{2}\right) \cdot T_{n-1} \\
& =\left(2+A_{d}+b \cdot A_{d-2}\right) \cdot T_{n}-\left(1+A_{d}+b \cdot A_{d-2}+(-b)^{d}\right) \cdot T_{n-1}
\end{aligned}
$$

where we have used the identity

$$
\begin{aligned}
A_{d} \cdot A_{d-2}-A_{d-1}^{2} & =\frac{-(-b)^{d-1}}{a^{2}+4 b}\left[\left(\frac{a+\sqrt{a^{2}+4 b}}{2}\right)^{2}+\left(\frac{a-\sqrt{a^{2}+4 b}}{2}\right)^{2}\right]+\frac{2(-b)^{d}}{a^{2}+4 b} \\
& =-(-b)^{d-1} \cdot \frac{a^{2}+2 b+2 b}{a^{2}+4 b}=-(-b)^{d-1} .
\end{aligned}
$$

Now, our theorem follows from formula (6).

\section{Competing interests}

The authors declare that they have no competing interests.

\section{Authors' contributions}

$\mathrm{XL}$ studied the Smarandache-Pascal derived sequences and proved a generalized conclusion. DH participated in the research and summary of the study.

\section{Acknowledgements}

The authors would like to thank the referee for carefully examining this paper and providing a number of important comments. This work is supported by the N.S.F. $(11071194,11001218)$ of P.R. China and G.I.C.F. (YZZ12062) of NWU.

\section{Received: 9 June 2013 Accepted: 23 July 2013 Published: 8 August 2013}

\section{References}

1. Smarandache, F: Only Problems, Not Solutions. Xiquan Publishing House, Chicago (1993)

2. Murthy, A, Ashbacher, C: Generalized Partitions and New Ideas on Number Theory and Smarandache Sequences, p. 79. Hexis, Phoenix (2005)

3. Rong, M, Wenpeng, Z: Several identities involving the Fibonacci numbers and Lucas numbers. Fibonacci Q. 45 164-170 (2007)

4. Yuan, Y, Wenpeng, Z: Some identities involving the Fibonacci polynomials. Fibonacci Q. 40, 314-318 (2002)

5. Tingting, W, Wenpeng, Z: Some identities involving Fibonacci, Lucas polynomials and their applications. Bull. Math. Soc. Sci. Math. Roum. 55(1), 95-103 (2012)

doi:10.1186/1687-1847-2013-240

Cite this article as: Li and Han: On the Smarandache-Pascal derived sequences and some of their conjectures. Advances in Difference Equations 2013 2013:240. 